\title{
I am struggling to understand why patients decide not to get the covid vaccine
}

\author{
As rates of omicron rise, an anaesthetist deployed to intensive care during covid surges describes \\ her anger and disappointment that people choose not to have the covid vaccine
}

I am a consultant anaesthetist. I work in a busy district general hospital, in an area badly affected by covid, serving a population whose vaccination rate is significantly lower than the national average. It is a population that is also socially and economically deprived with all the associated health effects. In the first two waves of the pandemic I worked in intensive care, and I will do so again within weeks.

In my career I have treated hundreds of patients who are in hospital because of choices they have made: smokers, skiers injured on the slopes, along with patients who have inserted various objects into orifices not intended for the purpose. I have never felt judgemental. I understand it is their right to choose, and while I may not agree with their choices, my job is to treat them with compassion, but not judgement. I have never found that difficult.

Until now. I am struggling to understand why patients decide not to get the covid vaccine. I see patient after patient on the labour ward, in emergency theatre, and in intensive care who have chosen not to have the vaccine. The reasons vary-from worry about long term effects, through fertility, to inaccurate ideas about microchips and government surveillance.

Almost exclusively those needing intensive care or dying with covid are young, healthy, and unvaccinated. Only a tiny proportion are vaccinated vulnerable patients with other underlying health conditions, or are partially vaccinated. This despite scientific research and real-world evidence showing that vaccination works. Vaccination offers us the sole opportunity to avoid further lockdowns and lead relatively normal lives. I find these self-destructive decisions incomprehensible.

There is also the question of free riding. The unvaccinated enjoy the benefits-social freedoms and much reduced risk of covid infection-because the majority of adults, and now adolescents, choose to take the vaccine.

A small number who get sick need our care. Some need to come to intensive care, or have their babies delivered early as they are too sick to remain pregnant. They require huge resources-the average stay in intensive care is two weeks, many stay for months, and some do not survive. The impacts on healthcare professionals include ongoing risks of being infected. Most frontline healthcare professionals had covid before the vaccines were available due to exposure at work. At best we got sick, at worst some of us died, and sadly, continue to die. Healthcare workers carry the emotional impact of caring for, and sometimes losing, young, previously healthy individuals who are critically unwell from covid, when this could have been avoided if they had had the vaccine. The physical and mental challenge of caring for these patients is huge: the numbers are still large enough to affect the care we want to give to non-covid patients. We cannot always proceed with elective surgery, or get an intensive care bed for an emergency patient who needs it for non-covid reasons.

We are tired. We are working at greater capacity than ever before and have been for nearly two years. I worry that this sustained pressure will break us. We will make mistakes, and this impacts everyone. It's already happening.

At present rate, we will never be able to clear the backlog. There is sustained pressure on our hospital wards and intensive care units. The waits will get longer, the service will at times fall short of the high standards we take for granted. For the first time in my professional life, I am angry with my patients who choose not to get vaccinated, and while I do not let it affect my patient care, I think it's affecting me.

I do not believe in forcing people to get the vaccine, and I feel uncomfortable that it is happening in the NHS. But equally, I am just so disappointed that so many people continue to choose not to take the vaccine. I can't understand it. Unlike the patients needing our care due to the ordinary choices they have made, choosing not to get vaccinated is different. It doesn't just affect the individual, it affects us all.

Competing interests: none declared

Provenance and peer review: not commissioned, not peer reviewed 